\title{
Ueber das
}

\section{Vorkommen von Klappenapparaten}

in den

Excretionsorganen der Trematoden

\author{
Karl KA M P MA N N \\ in Genthin. \\ Hierzu Tafel XIX und XX.
}

Während die Kenntnis von Klappen in den Excretionsapparaten der Cestoden auf einen Zeitraum von circa zwanzig Jahren zurückgeführt werden kann, ist bisher von derartigen Einrichtungen in den gleichnamigen Organen der Trematoden nichts bekannt geworden, und selbst die in jüngster Zeit auf diesem Gebiete veröffentlichten Arbeiten enthalten keine diesbezüglichen Angaben.

Der Schwerpunkt bei der Untersuchung der einzelnen Abschnitte des Excretionsapparates ist bei den Trematoden mit wenigen Ausnahmen und vielleicht mit Recht auf die Beschaffenheit derjenigen Theile gerichtet worden, welche die Arbeit der Aufnahme der Excretionsstoffe aus dem Körper bezw. dessen Parenchym übernommen hatten, während die leitenden und sammelnden Abschnitte nicht so eingehend in Beobachtung genommen wurden.

Nicht uninteressant und vor allen Dingen nicht ohne wissen- 
schaftlichen Werth dürfte die Thatsache sein, dass auch bei den Trematoden und zwar in den zuletzt erwähnten sammelndeu und leitenden Abschnitten derartige Klappen und klappenartig functionirende Linrichtungen vorhanden sind, welche in gleicher Weise wie bei höher organisirten Thier en dafür Sorge trag?n, dass unter normalen Verhältnissen diejenigen Stoffe, welche zur Ausfuhr aus dem Körper bestimmt sind, nicht in diejenigen Gebiete, aus denen sie kommen, zurückgelangen können.

Diese Einrichtungen finden sich bei einigen derjenigen Distomen, deren excretorische Apparate Sammelkanäle und eine Endblase besitzen; sie haben ihren Sitz an der Uebergangsstelle aus den Kanälen in die Blase.

Von nicht zu unterschätzender Bedeutung mag es sein, dass der Beweis für diese Behauptung nicht wie bei den Klappenapparaten der Cestoden fast nur allein durch die am toten Material gewonnenen mikroskopischen Präparate, sondern durch die Beobachtung ihrer Function am lebenden Thiere geführt werden kann.

Das Gebiet der auf Lösung dieser Frage gerichteten Untersuchungen erstreckte sich auf eine Reihe von Distomen, und wenn auch die Zahl der untersuchten verschiedenen Arten bei der unendlich grossen Menge, welche es von diesen überhaupt giebt, eine relativ kleine geblieben ist, so dürfte gegen etwaige dahin zielende Vorwürfe der positive Erfolg der angestellten Untersuchungen entschultigend eintreten; das Material zur Prüfung und Bestätigung meiner Angaben bei anderen Distomen bleibt für andere Forscher ein um so grösseres.

Bevor ich auf die einzelnen Methoden, auf die einzelnen Arten der untersuchten Thiere und die bei denselben festgestellten Einrichtungen eingehe, will ich, unter möglichster Vermeidung unnöthiger Ausdehnung, aus derjenigen Litteratur, welche für die in dieser Arbeit aufgestellte Frage in Betracht kommt, Nachstehendes hervorheben : 
SOMMER ${ }^{1}$ äussert sich in seinem Werk über Dist. hepaticum in Bezug auf den excretorischen Apparit u. a. pag. 586 wie folgt : "Die Wand des excretorischen Apparats wird von einer überaus feineu, structurlosen und elastischen Gewebsschicht gebildet, deren Inmenfläche nirgends eine Zellauskleidung zeigt, mithin jeglicher epithelialen Decke entbehrt. Wirkliche Klappenvorrichtungen an den Einschnürungsstellen des Stammes sind nirgends nachweisbar; ebenso fehlen dem letzteren auch alle Andeutungen vom Flimmerlappen, deren Anwesenheit für eine Anzahl anderer Distomen von den Forschern behauptet worden ist. Ein muskulöser Belag der Wand des Stammes, der ihm eigene Contractilität verleihen und den Charakter eines Expulsionsorganes geben könnte, ist nicht vorhanden. *

Dieser Autor sagt ferner pag. 586: «Eine in allen wesentlichen Theilen korrekte Schilderung des Organes ist endlich von $R$. LeUckaRt in dessen grossen Parasitenwerke gegeben worden. »

LEUCKART ${ }^{2}$ giebt uns über die in Frage kommenden Abschnitte des Excretionsapparates bei den Trematoden folgenden Aufschluss: "Um die Anordnung dieses Excretionsapparates im Allgemeinen kennen zu lernen, unterscheidet man daran am besten drei verschiedene Abschnitte: den Sammelraum mit seinem Porus, der die Absonderungsprodukte nach aussen bringt (die Endblase), das System der grösseren Gefässe, die vorzugsweise, wie es scheint, zur Fortleitung dienen, und die davon ausgehenden feinen Capillaren, die ganz ebenso, wie wir das für die entsprechenden Theile der Cestoden kennen gelernt haben, je in einen Flimmertrichter auslaufen und in functioneller Beziehung offenbar am wichtigsten sind. »

\footnotetext{
'Sommer. Die Anatomie des Leberegels Distomum hepaticum L. Zeitschrift für wissenschaftliche Zoologie. 1880.

${ }^{2}$ R. Leuckart. Die Parasilen des Menschen, I. Band, dritte Lieferung, II. Auflage, Seite 36.
} 
Von irgend welchen Klappen oder klappenartig functionirenden Einrichtungen im Bereiche der Endblase und der Sammelgefässe sagt LEUCKART nichts.

Loos ' bestätigt FraIPont's Angaben über den Bau der Excretionsorgane, richtet dabei aber sein Hauptaugenmerk auf die Flimmertrichter, also auf den Ursprung der Kanäle und erwähnt nichts über den Eintritt der letzteren in die Blase.

Loos hat u. a. Distomum clavigerum, Distomum endolobum, Distomum cygnoides, Distomum ovocaudatum und Distomum globiporum untersucht.

Fraipont ${ }^{2}$, dem wir die ergiebigsten Autklärungen über den Excretionsapparat der Trematoden und Cestoden in neuerer Zeit verdanken, hat bei dem Distomum squamula folgende Eintheilungen der kxcretionsorgane gemacht; er unterscheidet drei Partien: "la vésicule terminale, un système de gros canaux qui en partent et un système de fins canicules qui débouchent dans ces canaux et qui prennent leurs points d'origine dans de petits entonnoirs ciliés. »

Fraipont beschreibt sodann die einzelnen Abschnitte eingehend, und giebt zu den Beschreibungen die erklärenden Illustrationen. Er spricht bei der Beschreibung der grossen Gefässe von einer "Scheidewand " oder einem "Diaphragma", welche an der Bifurcationsstelle der grossen Leitungskanäle sich befinden; man sieht in der auf Tafel XVIII gezeichneten Figur 2 an der genannten Stelle einen Zipfel in den Haupt. stamm, welchen die beiden grossen bilden, hineinragen. Dieser Zipfel, falls er durch das ganze Lumen des Gefässes geht, kann eventuell als Verschlussventil aufgefasst werden, und auch als solches fungiren, immerhin aber erfüllt er nicht

${ }^{1}$ Beitrag zur Kenntniss der Trematoden. Zeitschrift tür wissenschaftliche Zoologie, 1885ั, pag. 405 .

${ }^{2}$ Jul. Fraipont.- Recherches sur l'appareil excreteur des Trematodes et Cestodes, Archives de Biologie, 1880, t. I. 
den Zweck, einen Abschluss zwischen Blase und zuführenden Gefässen herzustellen. Seine Function kann sich lediglich darauf beschränken, den einen Kanal zu schliessen, während sich der andere seines Inhalts entlecigt und ungekehrt; einen Abschluss der Base gegen Rückstauung ihres Inhalts in die Gefässe zurück vermag er nicht herzustellen.

Fraipont sagt pag. 425: «Au point, où a lieu la bifurcation du tronc commun d'origine (Fig. 12), on remarque, chez les jeunes Distomes, une cloison ou un diaphragme, sur lequel nous ne pouvons insister, étant donnée notre ignorancè du mode de développement de l'appareil. »

Dass FraIPont es besonders betont, dass man bei den jungen Thieren diese Beobachtungen machen kann, hat, wie wir später sehen werden, seine wohlbegründete Berechtigung.

Derselbe Forscher schenkt in einer zweiten Arbeit ${ }^{1}$ dem Eintritt der grossen Gefässstämme in die Endblase eingehendere Beachtung. Bei diesen vergleichenden Untersuchungen der Excretionsapparate der Cestoden und Trematoden hat er das Distomum divergens als Untersuchungsobjekt gewählt und bei diesem festgestellt, dass die genannten Gefässstämme nicht direct in die Blase an deren Wand einmünden, sondern noch eine gewisse Strecke in das Lumen der Blase hineinragen. Dieses Endstück der Gefässstämme ist nach FRAIPONT im Innern mit Cilien dicht besetzt. Er sagt pag. 2: « Ces deux trones sont plus rapprochés de la face dorsale que de la face ventrale du ver. Leur mode d'insertion sur la vésicule est particulier. Ils ne débouchent pas directement dans celle-ci, mais ils y pénètrent jusqu'à une certaine profondeur en conservant leur diamètre extérieur. Le bord libre de leur orifice et leur paroi interne sont complètement couverts de cils vibratiles. Cette

${ }^{1}$ Recherches sur l'appareil excréteur des Trematodes et Cestodes. Archives de Biologie, 1881, II. 
couche ciliaire s'étend dans le canal jusqu'à une certaine distance en dehors de la vésicule. »

Auch über diese Beobachtungen sind von Fraipont Zeichnungen beigegeben, indessen ist aus denselben, soweit sie die Figuren 3 und 5 betreffen, wo die Objekte körperlich gedacht sind, nicht mit absoluter Sicherheit zu erkennen, ob diese Fortsetzungen der Gefässstämme nicht vielmehr als Verschluss der Blase gegen das Gefäss dienen sollen.

LANG ${ }^{1}$ bezeichnet in den allgemeinen Bemerkungen über Plathelminthen das sogen. Wassergefässsystem, für diese sehr charakteristisch, " als ein System feiner, einerseits sich im Mesoderm verästelnder, andererseits nach aussen mündender Kanäle; " und weiter unten ${ }^{2}$ : " Auch bei den Trematoden finden wir typisch zwei Längsstämme, die entweder durch Vermittlung einer gemeinsamen contractilen Endblase von sehr verschiedener Grösse am hintern Körperende nach aussen münden (Distoma) oder getrennt durch zwei erweiterte Endstücke im vordern Körpertheile dorsalwärts sich nach aussen öffnen.

MONTECELLI ${ }^{3}$ constatirt bei seiner anatomisch-histologischen Beschreibung einer ganzen Anzahl Distomen, die von Pintwer, Fraipont, Loos und andern angegebenen Einrichtungen der Excretionsorgane; bezüglich der Abgänge aus den Kanälen in die Blase macht er keine Angaben.

NoAcK " macht bezüglich des Excretionsapparates bei dem Dist. clavigerum dieselbe Eintheilung wie Fraipont bei Dist. squamula.

Die von NoAcK beschriebenen beiden Hauptstämme «münden

${ }^{1}$ LANG. Lehrbuch der vergl. Anatomie. 1889, pag. 135.

${ }^{2}$ Lang. Lehrbuch der vergl. Anatomie. 1881, pag. 152.

${ }^{3}$ Studii sui Trematodi endoparassiti di Fr. Sav. Monticelli. Jena, 1893.

+ Die Anatomie und Histologie des Distomum clavigerum Rud. Von Ernst NoACK, 1892. 
in den auf ihrer Seite gelegenen Schenkel der Blase und zwar am vordern Ende, meist ein wenig seitlich, ohne sich dabei merklich zu erweitern.

Der Centraltheil, also die Blase, stellt einen länglichen, sackartigen Behälter dar, welcher sich vorne in zwei meist ungleich lange Schenkel spaltet.

Von einem Verschlussapparat oder dergleichen an der Eintrittsstelle der Hauptstämme in die Blase hat NoAcK nichts erwähnt und anscheinend auch nichts gesehen, denn die seiner Arbeit beigefügte Zeichnung (Taf. I, Fig. 2) lässt die genannten Stämme in keiner Weise gehindert in die Blase eintreten.

BRAUN $^{1}$ giebt dem Excretionsapparat der meisten Distomen die gleiche Eintheilung, ohne auch nur bei einem einzigen derselben das Vorhandensein von Klappen oder dergleichen zu besprechen. Ueber den Bau der Sammelröhren und der Blase sagt Braun Folgendes : * Freilich ist es in solchen Fällen schwer oder unmöglich, die zipfelförmigen Anhänge der Endblase gegen die sammelröhren abzugrenzen, namentlich wo letztere das gleiche Caliber wie die Excretionsblase haben oder sich an ihrem hinteren der Einmündungsstelle zugerichteten Ende mehr oder weniger erweitern. Vielleicht wird einmal eine genauere histologische Analyse der Wandung Unterschiede erkennen lassen, wie z. B. Fraipont angiebt, dass die Endstücke der Sammelröhren von Dist. divergens Rud., die bei dieser Art eine Strecke weit in das Lumen der wenig entwickelten Zipfel der Endblase frei hineinragen, Wimpern tragen, sonst aber wimperlos sind. Bis wir Genaueres wissen, müssen wir nach anderen, weniger sicheren Anhaltspunkten suchen ; möglicherweise sind solche in dem verschiedenen Inhalte der Sammelröhren und der Excretionsblase gegeben. »

In den Neapeler Jahresberichten finde ich in den Jahr-

${ }^{1}$ Brons's Klassen und Ordnungen des Thierreichs. IV. Band, 1892, p. 642. 
gängen 1879 bis 1892 keine Angaben, welche darauf hinweisen, dass von Zoologen der in Rede stehenden Frage durch Veröffentlichung von Untersuchungsbefunden anderweitig näher getreten sei.

Bevor ich zu der Darstellung meiner Beobachtungen übergehe, will ich kurz über die angewandten Untersuchungsmethoden berichten.

Dass für die Untersuchungen der in Frage kommenden Organe das Alter und die Geschlechtsreife der Thiere eine grosse Bedeutung haben, ist schon in Bezug auf letztere bei Beschreibung der einzelnen Untersuchungsobjekte erwähnt worden; je mehr der Uterus mit Eiern gefüllt, um so schwerer ist eine Untersuchung,

Da nun die Zunahme der Eier mit dem zunehmenden Alter wenigstens eine Zeit lang gleichen Schritt zu halten pflegt, so ist es einleuchtend, dass die jungen Thiere zur Untersuchung ein passenderes Material bieten, als die alten. Auf diesen Umstand hat auch Fraipont ${ }^{1}$ hingewiesen und deshalb die jungen Distomen zur Untersnchung empfohlen, um die von ihm beobachteten Scheidewände in den Sammelkanälen und Fortsetzungen mit ihrer Wimperung in der Endblase sehen zu können.

Dasselbe gilt für die von mir untersuchten Distomen.

Bei der Untersuchung lebender Thiere ist ferner darauf zu achten, dass die Ortsbewegungen derselben unter dem Deckglas möglichst eingeschränkt werden, während die Thätigkeit der Organe ungestört bleibt; man erreicht dieses nach meinen Erfahrungen am besten, indem man die Thiere mit einem kleinen Deckglase direkt belegt, ohne an letzteres Wachsfüsse oder dergleichen Unterstützungen anzubringen; von Zeit zu Zeit setzt man dem Präparat eine Wenigkeit physiologischer

1 L. c., pag. 42 כ. 
Kochsalzlösung zu. Bei dieser Behandlung bleiben die Thiere ziemlich auf demselben Flecke liegen, sie werden ausserordentlich übersichtlich und bleiben 2-3 Stunden am Leben.

Die auf diese Weise behandelten Thiere zeigen eine sehr deutliche Blase, diese füllt sich unter dem Deckglase, ihre Contouren treten scharf hervor, ohne dass die zuführenden Sammelkanäle ihre Dicke oder Fülle ändern; bei Thieren, welche man unter Deckgläsern, welche Wachsfüsse tragen, untersucht, kann man diese wichtigen Beobachtungen nicht machen, die Thiere haben hier mehr Spielraum sich zu bewegen, den Körper zusammenzuziehen und auszudehnen und bei diesen Bewegungen die Blase zu entleeren.

Um gutes Material für Schnitte zı erhalten, muss man nach meinem Dafürhalten die Thiere nicht unter dem Deckglase töten; letzteres erscheint mir für Gewinnung guten Materials geradezu hinderlich. Den Druck des Deckglases hält das lebende Thier für die Zeit der Untersuchung aus, der elastische Körper und die Muskeln leisten dem Druck von oben Widerstand und weichen nur dem lange anhaltenden Druck; auf die toten Organe aber übt das Gewicht des Deckglases schon einen nachhaltigen Einfluss aus, es drückt die zarten Klappen fest an die Blasenwand, es verändert ihre normale Lage und Stellung.

Diesem Umstande allein glaube ich es zumessen zu müssen, dass ich die im Leben bei Dist. mentulatum so deutlich wahrgenommenen Klappen an Schnitten nicht habe wieder finden können.

Das mir zur Verfügung stehende Material war unter dem Deckglase abgetötet worden; neues konnte ich trotz ausgedehntester Bemühungen nicht erlangen.

Dass für die Feststellung von Klappen am toten Material fast nur Längsschnitte angefertigt wurden, darf wohl kaum besonders hervorgehoben werden, die Nothwendigkeit hierfür 
liegt in der anatomischen Anorenung der Endblase und der Sammelkanäle zum Körper.

Bezüglich der Tötungs- und Färbemethoden sei kurz Folgendes erwähnt: Die Abtötung geschah mit Sublimat, mit Alkohol und mit Chrom-Osmiumessigsäure.

Distomum isostomum und Dist. cirrigerum habe ich mit Pikrokarmin, Alaunkarmin, Eosin, Hämatoxylin und mit der Doppelfärbung Eosin-Hämatoxylin gefärbt; auch wurde Boraxkarmin versucht, die übrigen Thiere meistens mit dem sich als am geeignetsten zeigenden Pikrokarmin tingirt.

Bei Gelegenheit des zoologischen Praktikums fand ich in den Muskeln eines Flusskrebses Parasiten; es war das Distomum isostomum, vertreten durch wenige Exemplare.

Der Parasit zeigte sich als ein zur Beobachtung und Untersuchung im lebenden Zustande äusserst geeignetes Objekt, und wenn von Professor Schneider und Dr. GafFron diese rühmliche Eigenschaft besonders hervorgehoben wird ', dann kann ich mich dieser Ansicht nur im weitesten Sinne anschliessen.

Das Bild, welches namentlich der Excretionsapparat mir bot, war überraschend; es zeigte sich am lebenden Thiere ein Klappenapparat zwischen der Endblase und den Sammel. kanälen, welcher über alles Erwarten vollkommen und exact functionirte.

Bei keiner Art der nachher durch mich untersuchten Distomen habe ich über den ganzen Verlauf des unendlich fein und weitverzweigten Kanalsystems einen so überaus klaren Ueberblick gewinnen können, als bei diesem Thiere.

Aus diesem Grunde habe ich auch Veranlassung genommen, in Fig. 1 ein Uebersichtsbild des ganzen Apparates, so wie ich ihn nach dem Leben gezeichnet habe, beizufügen.

${ }^{1}$ Gaffrox. Zum Nervensystem der Trematoden. Zool. Beiiräge. 
Die baumartig verzweigten kleinsten Kanälchen und die grösseren Kanäle fliessen jederseits in einen Hauptkanal zusammen, von diesem bleibt der vordere Hauptkanal einstämmig, während man an dem hintern eine Trennung in zwei ziemlich gleich starke und gleich lang nach hinten verlaufende Stämme wahrnehmen kann.

Gleich hinter dem Bauchsaugnapf treten die Hauptstämme von vorne und vou hinten in ein ziemlich auffällig erweitertes, fast im rechten Winkel nach innen ziehendes Gefäss. Dieses kann kaum als ein Kanal bezeichnet werden, es hat eine länglich-ovale Form und stellt ein Sammelbecken dar. Aus diesem Sammelbecken führt ein kurzer enger Kanal in die eigentliche Endblase; an der Mündung dieses Kanals in die Blase befindet sich eine Klappe.

Wir sehen bei dem unter dem Deckglase liegenden Distomum isostomum die Blase sich mit Inhalt füllen, der Excretionsporus öffnet sich bisweilen, es tritt Blaseninhalt nach aussen; in diesem Augenblick beobachten wir nun weiter, dass die vor der Mündung der vorhin erwähnten Kanäle befiudlichen Klappen sich jetzt an die Blasenwand anlegen, das Zngangsrohr verschliessen und eine Rückstauung in die Sammelbecken verhindern.

Während nun die Klappen zwischen den Kanälen und der Blase einen Verschluss herstellen, füllen sıch wieder die Sammelbecken, um, nachdem der Druch der Blase aufgehört hat, sich wieder zu entleeren. So bleibt Füllung und Leerung einerseits der Blase, andererseits der Sammelbecken stets in abwechselnder Reihenfolge in Betrieb; der Apparat functionirt tadellos. (Fig. 2.)

Die an dem lebenden Objekt gemachten Beobachtungen können an den von diesem Thiere angefertigten Schnitten bestätigt werden, man sieht die Klappen als selbsständige Gebilde durch mehrere schnitte hindurch; man kann ihren 
Verlauf, ihre Stärke und Anordnung beobachten. Auch gelingt es bei diesem Objekt Schnitte anzufertigen, auf welchen beide Klappen (Fig. 3, 4, 5) nachzuweisen sind; bei allen anderen Arten, welche ich untersuchte, ist mir solches nur mangelhaft oder gar nicht geglückt.

Die bei Dist. isostomum gewonnene interessante Beobachtung veranlasste mich, da das vorhandene Material verbraucht war, mir neues zu verschaffen. Diesem Verlangen stellten sich indessen grössere Hindernisse, als zu erwarten war, entgegen. Unter circa 1400 Krebsen, welche ich mir aus Ost-Preussen, Schlesien, Posen, Brandenburg und Mecklenburg habe schicken lassen, habe ich nur ein Thier gefunden, welches mit Dist. isostomum behaftet war.

Die Untersuchungsresultate hinsichtlich des Excretionsapparates waren genau dieselben und bestätigten einwandsfrei das Vorhandensein von Klappen.

Die Auffindung dieser Klappe bei Dist. isostomum veranlasste mich, bei einer Anzahl anderer Distomen mit Endblase und Kanalsystem nach ähnlichen Einrichtungen zu suchen.

Ichuntersuchte zu diesem Zwecke folgende Arten :

$\begin{array}{lll}\text { 1. } & \text { Distomum mentulatum Rudolphi. } \\ 2 . & \text { cirrigerum Bær. } \\ 3 . & \text { assula Dujardin. } \\ 4 . & \text { signatum Rudolphi. } \\ 5 . & \text { naja Rudolphi. } \\ 6 . & \text { } & \text { triganocephalum. } \\ 7 . & \text { crassicolle. } \\ 8 . & \text { clavigerum Rudolphi. } \\ 9 . \quad \text { } & \text { endolobum Dujardin. }\end{array}$

Unter den genannten Distomen fanden sich Klappen von dem bei Dist. isost. beschriebenen Bau nur bei Dist. mentulatum.

Dieses Thier eignete sich, da es in den vorhandenen 12 
Exemplaren durchweg eifrei war, zur Beobachtung im lebenden Zustande ganz besonders gut.

Das lange schmale Thier ist mit einer stimmgabelförmig gestalteten, bis ans vordere Ende des Thieres reichenden Blase ausgestattet. (Fig. 6.)

Die auf den Darmschenkeln liegenden Kanäle und Sammelröhren begleiten die Blase in ihrem ganzen Verlauf, bald dicht an diese, bald weiter an den Körperrand herantretend, in wellenfôrmigen Windungen. Die Kanäle vereinigen sich etwa in der Mitte der Körperlänge; hier bilden 2, 3 und mehr Gefässe, von denen konstant zwei grösser, die übrigen wesentlich kleiner sind, einen gcmeinsamen Stamm, welcher wieder ähnlich wie bei Dist. isostomum beckenartig sich erweitert, sich nach kurzem Verlauf der Blasenwand zuwendet und, diese von vorn nach hinten begleitend, sich in die Blase ergiesst. (Fig. 7, 8, 9.)

Hier bilden wieder Blasen- und Gefässwand eine Klappe, deren Bewegungen man deutlich beobachten kann. Bei dem Strudeln der aus dem Sammelrohr in die Blase fliessenden Excretionsflüssigkeit hebt sie sich mehr oder weniger von der Blasenwand ab, bei stärkerer Füllung der Blase tritt sie näher an die Wand heran, und bei Fntleerung der Blase durch den Excretionsporus legt sie sich fest vor die Oeffunng, oder so fest an die Wand, dass nur wenig Flüssigkeit in die Blase gelangen, erst recht aber nicht aus der Blase in die Kanäle zurückstauen kann.

Die Mündung des Sammelrohres ist nicht immer an einer bestimmten Stelle zu suchen, sie zeigt sich einmal näher der Gabelung der Blase, einmal näher an das vordere knde der Blasenschenkel verlegt, immer aber ist sie nur einmal auf jeder Seite vorhanden, und immer tritt das Sammelrohr von der äusseren Seite des Blasenschenkels her in die Blase ein.

Ich habe die Thiere stundenlang unter dem Deckglase liegen 
gehabt, während des Beobachtens fülite sich die Blase mit Excretionsstoffen und wurde mit zunehmender Füllung übersichtlicher. Mit zunehmender Füllung verminderte sich zwar der Zufluss, verlangsamte sich auch, bis endlich die Blase sich zusammenzog und ihren Inhalt durch den Excretionsporus entleerte ohne Rückstauung in das Sammelrohr.

Tötet man ein unter dem Deckglas liegendes Dist. mentulatum mit Sublimat, dann sieht man eine kurze Weile den Austritt der? Excretionsstoffe in die Blase continuirlich erfolgen, plötzlich setzt die Thätigkeit aus, die Klappe schwingt langsam ein paar mal hin und her, legt sich dann an die Wand an und bleibt hier liegen, wenn der Tod eingetreten ist.

Trotzdem die Klappen und ihre Functionen am lebenden Thier bei starker Vergrösserung deutlich wahrzunehmen und eingehender Beobachtung zugänglich sind, ist es mir nicht gelungen, dieselben an Schnitten nachzuweisen. Ich habe nicht $\mathrm{zu}$ ermitteln vermocht, wesshalb ich die Klappen am toten Material nicht wiederfinden konnte, ich vermuthe aber dass die Behandlungsmethode auf die überaus zarten und feinen Klappen einen derartig ungünstigen Einfluss ausgeübt hat und dass vor allen Dingen das Dichranlegen der Klappen an die Blasenwand eine Grenze zwischen beiden nicht hat feststellen lassen. Die Sammelröhren sind auf den Schnitten wohl zu finden, ihr Lintritt in die Blasenwand dagegen nicht.

Die negativen Befunde bei den von Dist. mentulatum angefertigten Schnitten sind indessen nicht im Stande, die Beobachtungen der Klappen am lebenden Thier in Frage zu stellen.

Ein anderes Untersuchungsobjekt fand sich in Dist. signatum, aus dem Oesophagus der Ringelnatter entnommen.

Dieses durchweg zur Untersuchung im lebenden Zustande sehr geeignete Thier zeigt eine sehr grosse, fast über die ganze hintere Körperhälfte sich ausdehnende Endblase (Fig. 10) von sehr geringem dorsoventralem Dürchmesser. 
Die zahlreich vorhandenen Uterusschlingen, welche mit Eiern gefüllt sind, drücken die Blasenwandungen bei ihren Bewegungen gegeneinander und gegen die Rückenfläche des Thieres. Hinter dem Bauchsaugnapf sieht man die grossen Kanäle von jeder Seite der Mitte zustreben (Fig. 10) und es macht den Eindruck, als ob sich die Kanäle hier zu einem gemeinschaftlichen kleinen Sammelbecken vereinigen, um aus diesem in die Blase zu münden.

Der Austritt des vermuthlich gemeinsamen Kanals in die Blase habe ich trotz sorgfältigster Beobachtung nicht ermitteln können; auch die Schnittserien haben mich bei den darauf gerichteten Nachforschungen im Stich gelassen.

Die negativen Resultate der Untersuchungen von Dist. signatum haben sich noch bei anderen Distomen-Arten wiederholt. Bei Dist. naja, Dist. triganocephalum, Dist. crassicolle haben die von mir angestellten Untersuchungen zu keinem definitiven Abschluss über die Verhältnisse zwischen den Sammelkanälen zur Endblase geführt; die Schuld hierfür war einzig wohl nur in dem Umstande zu suchen, dass alle diese Arten zur Zeit der Untersuchung übermässig mit Eiern gefüllt waren und durch die Anwesenheit der zahlreichen Eier eine genaue Feststellung der that:ächlichen anatomischen Einrichtung der excretorischen Endorgane unmöglich gemacht wurde.

Günstiger sind meine Untersuchungen bei Distomum cirrigerum, Distomum clavigerum und Distomum endolobum ausgefallen.

Bei diesen Thieren finden sich zwar nicht selbstständige Klappen, wie wir sie bei Dist. isostomum und Dist. mentulatum kennen gelernt haben, es kommt jedoch an der Einmündungsstelle der Sammelröhren in die Endblase eine Einrichtung zu Stande, welche in gleicher Vollkommenheit einen Abschluss beider Abschnitte gegen einander zu bewirken vermag.

Ich werde dieselbe zunächst von Dist. cirrigerum beschreiben. 
Unter der grossen Anzahl von Krebsen, welche ich mir zur Beschaffung von Dist. isostomum hatte kommen lassen, fanden sich zwei Thiere, welche ein anderes Distomum und zwar das Distomum cirrigerum beherbergten. Diese Parasiten waren aber nicht, wie Dist. isostomum frei lebend in den Krebsen anzutreffen, sondern encystirt; ich fand dieselben etwa $25 \mathrm{Stü} \cdot \mathrm{k}$ fest im Muskelfleisch sitzend; ihre Entfernung aus den Cysten bereitete einige Schwierigkeiten.

Während die von Dist. isostomum gefundenen Exemplare ohne Eier angetroffen wurden, waren im Dist. cirrigerum die Uterusschlingen mit solchen sehr stark gefüllt. Dieser Umstand erschwerte mir die bei diesem Thiere angestellten Nachforschungen über den anatomischen Baı der Excretionsorgane.

Dieser ist von jenem des Dist. isostomum insofern wesentlich verschieden, als ihm die Sammelbecken fehlen und auch die Anordnung der Sammelkanäle eine andere ist Wir sehen zwei Hauptkanäle von vorn herkommend, ziemlich weit vom Blasengrund seitwärts in die Blase einmünden (Fig. 11). Vor ihrem Eintritt in die Blase zeigen die Kanäle zwar eine kurze Strecke lang eine Erweiterung, aber so beckenartig, wie sie bei Dist. isostomum im Totalpräparat zu sehen ist, ist dieselbe keineswegs.

Nach der Blase zu verjüngen sich die Kanäle zusehends; sie treten unter sehr spitzem Winkel in die Blase ein (Fig. 12). Blasenwand und die dieser zugekehrte Kanalwand verschmelzen zu einer langen, dünnen Membran (Fıg. $13 a$ ), welche als feines Häutchen die Blasenwand nach hinten hin eine Strecke lang begleitet.

Am lebenden Thier sieht man nun, dass sich dieses Häutchen bei Kontraction der Blase gegren die Wand derselben legt und so einen Verschluss der Samenröhren herbeiführt; nach erfolgter Entleerung der Blase löst sich das Häutchen wieder und der Zufluss aus den Kanälen ist wieder frei. 
Die von diesem Thiere angefertigten Schnitte (Fig. 12 u. 13) bestätigen den am lebenden Thiere gemachten Befund.

Wenngleich das Resultat der am Dist. cirrigerum gemachten Beobachtungen nicht so günstig ausgefallen war wie bei dem Dist. isostomum, indem nicht so deutlich functionirende Klappen gefunden wurden, so zeigte doch der Befund am lebenden und toten Thier, dass bei den Beziehungen zwischen Blase und Sammelrohr dasselbe Prinzip herrscht, nämlich er-tere gegen letzteres unter gewissen Bedingungen zu verschliessen; es kann die aus Blasen- und Kanalwand verschmolzene zarte Haut als ein « klappenartig functionirender Apparat » bezeichnet werden.

Das Dist. clavigerum zeigt im Totalpräparat die in Fi.. 14 angegebene Beschaffenheit der Endblase nebst den in diese einmündenden Sammelkanälen. Der Eintritt der Kanäle an der Aussenseite der Blasenschenkel ist ganz charakteristisch und typisch (Fig. 15), nur ausnahmsweise kommen Abweichungen vor.

Am lebenden Thier sieht man dies Sammelrohr scheinbar in das Lumen des Blasenschenkels hineinragen (Fig. 15), diẻses Bild bekommt mit dern von FRAIPONT beobachteten Verhalten bei Dist. divergens ' überraschende Aehnlichkeit. Man beobachtet sehr gut, dass aus dem Sammelrohr Excretstoffe, kleine hellgraue, auch glashelle Körnchen und flüssige Massen in die Blase hineingelangen, und dass bei einer Kontraction der Blase keine Theile dieser Massen wieder in das Rohr zurücktreten.

Ein Flimmern habe ich an dem Eintritt des Sammelrohres in die Blase nicht beobachtet.

Die von Dist. clavigerum angefertigten Schnitte geben über die am lebenden Thier gemachten Beobachtungen einige Aufklärung.

Die in den Figuren 16, 17, 18 wiedergegebenen Schnitte

1 L. c., page 2, PI. I, Fig. 3. 
lassen auf das deutlichste erkennen, dass der Eintritt der Sammelröhren nicht wie NoAcK ' angiebt " am vorderen Ende, meist ein wenig seitlich », sondern ganz bedeutend weit nach hinten und an der Seite der Blasenschenkel einmünden.

Diese typische Art der Finmündung haben wir schon hervorragend bei Dist. cirrigerum kennen gelernt. Figur 16 zeigt den Eintritt in den linken Blasenschenkel, während die Figuren 17 und 18 den Schnitt durch den rechten Blasenschcnkel erkennen lassen. Die auch hier wiederverschmolzene Wand der Blase und des Kanals setzt sich als eine dünne feine Membran, als eine Haut in die Blase fort und fungirt auch hier wieder als Schliessapparat zwischen Blase und Sammelrohr.

Aus diesen Ergebnissen an den Schnitten erklärt sich auch die am lebenden Thier wahrgenommene Erscheinung, als ob das Rohr sich in toto ins Blasenlumen fortsetzte. Die gemeinsame Blasen- und Gefässwand täuschte am lebenden Objekt dem Beobachter ein Bild vor, welches den Anschein hatte, als ob das ganze Rohr eine Strecke weit hineinrage. Ich muss nach diesen meinen Beobachtungen annehmen, dass FraIPONT sich in Bezug auf diesen Punkt bei Dist. divergens geirrt haben kann; leider habe ich mir keine Exemplare von Dist. divergens beschaffen können, um event. mir über die Frage volle Aufklärung zu verschaffen. Der Verschluss durch die genannte dünne Membran wird bei Kontractionen der Blase genau in derselben Weise herbeigeführt, wie wir es bei Dist. cirrigerum gesehen haben. Würde bei Dist. clavigerum ein einfaches Hervorragen des Sammelrohrs in die Blase, wie FraIPont es bei Dist. divergens beschreibt, vorhanden sein, das Lumen des Rohrs nach der Blase zu offen stehen, dann stände ja dem Rücktritt des Blaseninhalts in das Sammelrohr kein Hindernis entgegen. Dass aber ein Verschluss bezweckt wird, zeigen 
uns die Excretionsorgane der Cestoden und vieler höherer Thiere; es kann demnach auch wohl angenommen werden, dass die anatomischen Einrichtungen bei dem Dist. divergens ganz ähnliche sind, wie ich sie bei dem Dist. clavigerum gefunden habe.

Würden die Sammelröhren sich, wie NoAck meint, bei ihrer Mündung in die Blase, wenn auch unmerklich, erweitern, dann würde uns nicht nur die Grenze zwischen Blase und Sammelrohr fehlen, es würde die einmal ausgeschiedene Excretionsflüssigkeit in die Kanäle zurückstauen, diese mehr oder weniger erweitern und die Aufnahme der Excretstoffe in die Kanäle nicht unwesentlich stören.

Ganz ähnlich, wie bei dem Dist. clavigerum liegen die Verhältnisse bei Dist. endolobum.

Wir sehen eine anders gestaltete Blase, es fehlen die nach vorn sich ausdehnenden Schenkel, die Blase bildet einen Cylinder, an seinen beiden Seiten befinden sich die Mündungsstellen der Sammelröhren.

Die beigegebenen Figuren mögen uns orientiren.

Figur 19 und 20 zeigt schematisch die Blase mit den Sammelkanälen; Figur 21 lässt beide Sammelkanäle erkennen, während die Figuren 22 und 23 die Einmündung des Sammelrohres in die Blase auf ihrer linken Seite wiedergeben. Wieder sehen wir die gemeinsame Wand der Blase und des zuführenden Sammelrohrs einen Verschluss gegen das Kanallumen bilden; wieder zeigt sich das Prinzip ausgesprochen, den Austritt der Excretstoffe aus den Kanälen in die Blase frei zu gestatten, deren Rücktritt aber aus der Blase in das Kanalsystem zu verhindern.

Fassen wir, am Schlusse der Darstellung unserer Betrachtungen angelangt, noch einmal die Ergebnisse derselben zusammen, so haben wir die Thatsache festzustellen, dass bei sämmtlichen zur Beobachtung gelangten Distomen mit excretorischer 
Endblase die Sammelgefässe seitlich in die letztere eintreten, welche Anordnung allein einen klappenartigen Verschluss an der Mündungsstelle möglich macht.

Die Verschlussvorrichtungen sind auf zwei verschiedene Weisen zur Ausbildung gelangt.

Bei Dist. isostomum und Dist. mentulatum treten die Sammelröhren unter einem annähernd rechten Winkel an die Blase heran und in diesem Falle werden sie bei den Kontractionen der Endblase durch frei in den Hohlraum der letzteren hineinschwingende Klappen verschlossen.

Bei Dist. cirrigerum, Dist. clavigerum und Dist. endolobum hingegen bilden die Sammelröhren mit der Wand der Endblase einen sehr spitzen Winkel, wobei die der Blase zugekehrte (mediane) Wand des Sammelrohrs mit der Blasenwand zu einem am Grunde dickeren, gegen die Mündungsstelle des Rohres sich verdünnendem Häutchen verschmilzt, welches bei der Kontraction der Endblase durch den Gegendruck des Inhalts derselben vor die Mündung gelegt wird und diese verschliesst. 
TAFEL XIX

Figurenerklaterung

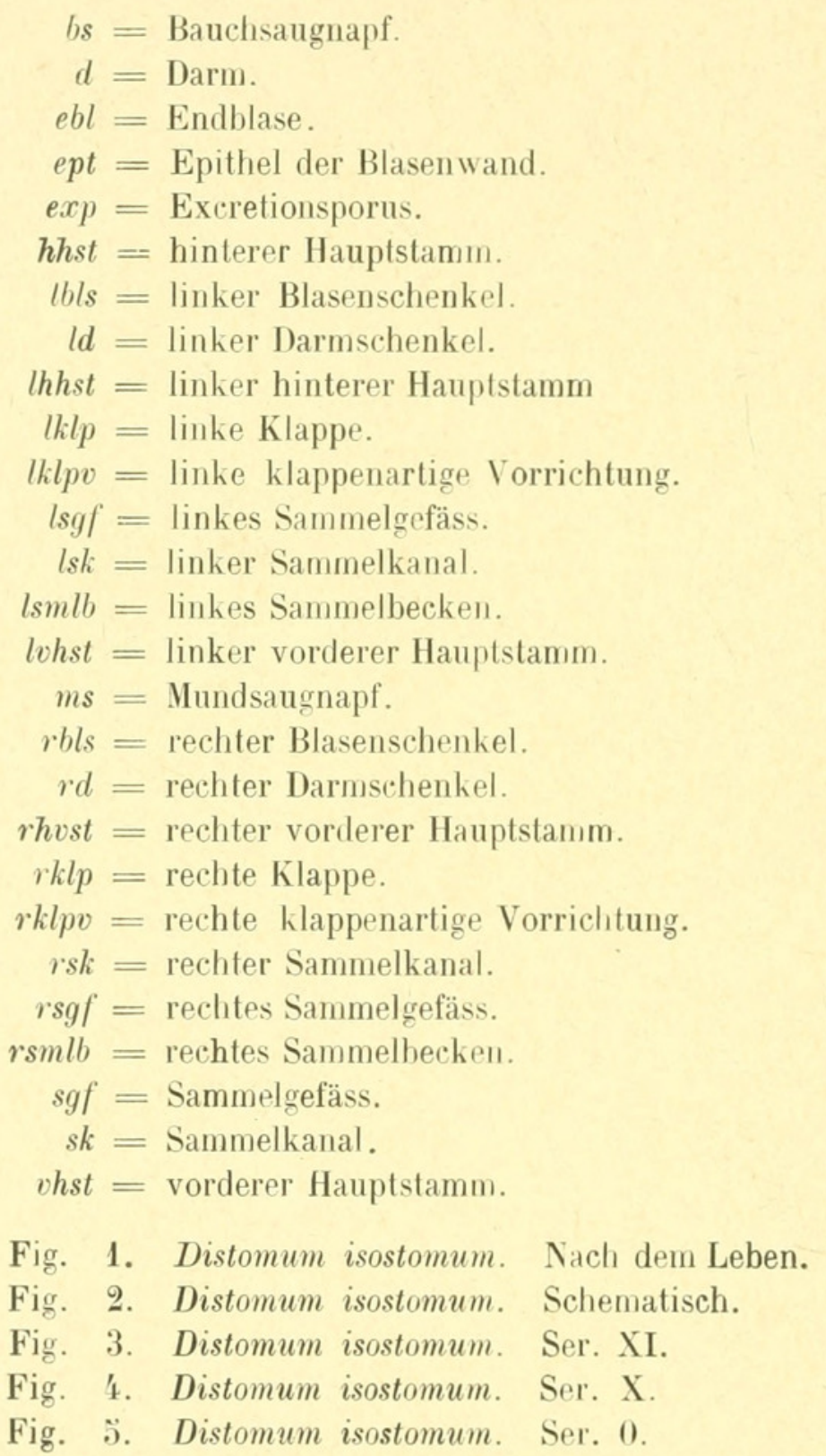



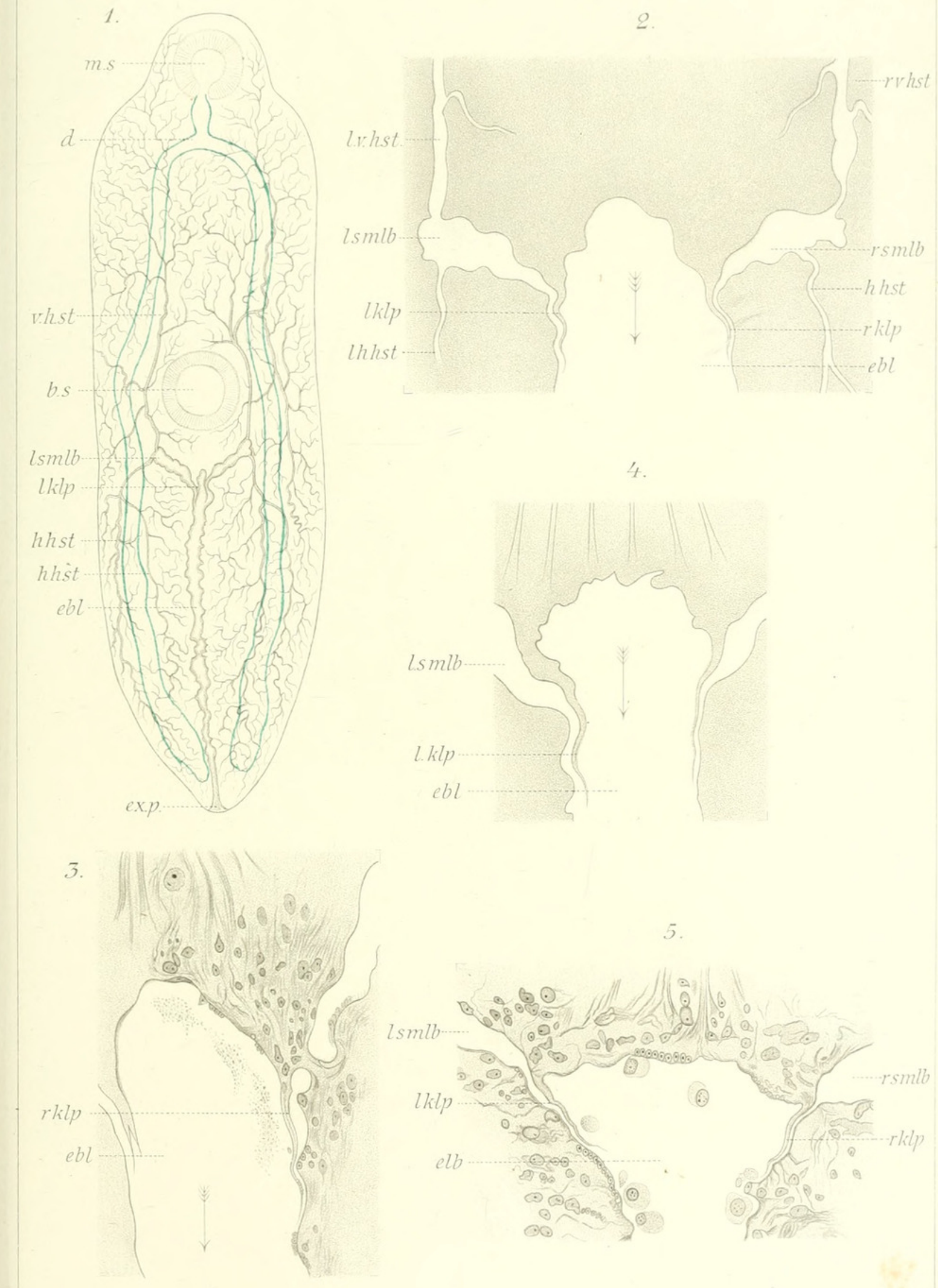


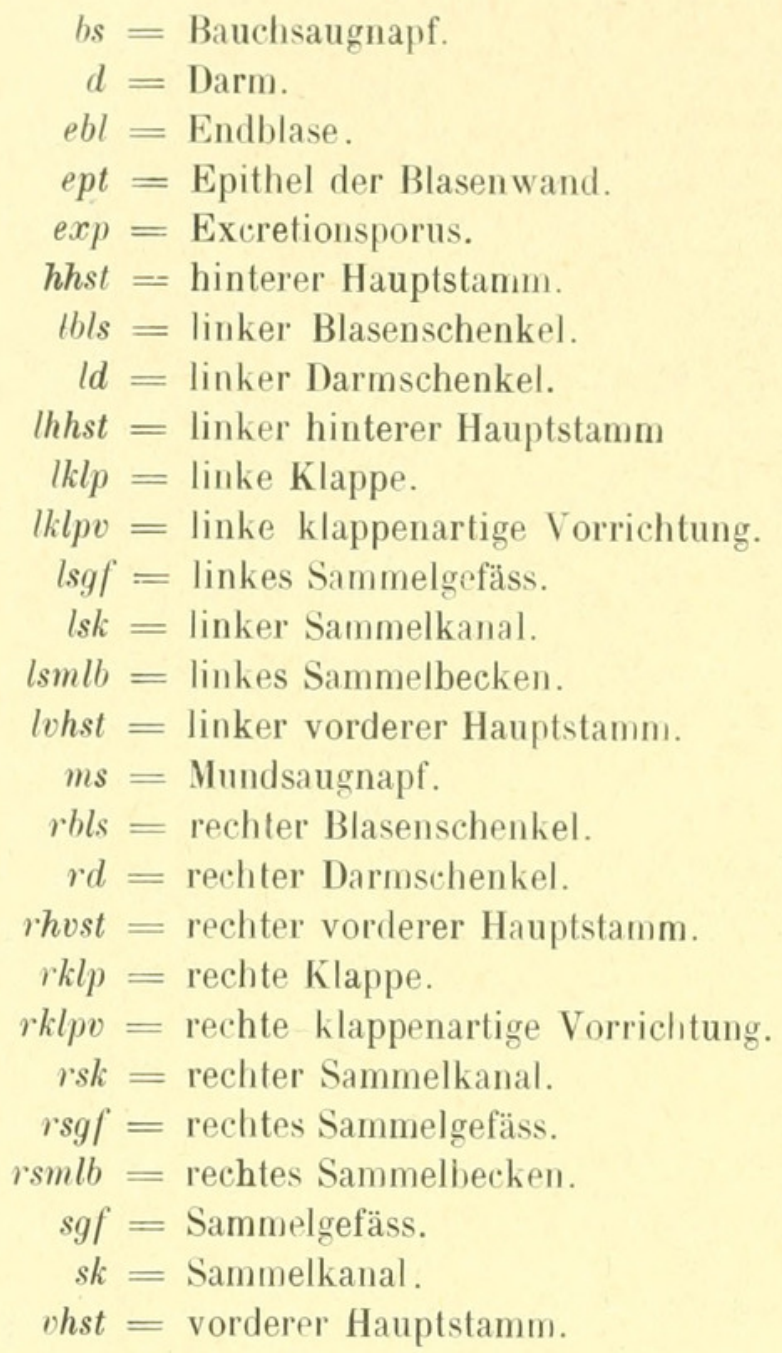

Fig. 6-9. Distomum mentulatum. Nach dem Leben.

Fig. 10. Distomum signatum. Nach dem Leben.

Fig. 11. Distomum cirrigerum.

Fig. 12. Distomum cirrigerum. Ser. V $b$.

Fig. 13. Distomum cirrigerum. Ser. VIII.

Fig. 14. Distomum clavigerum. Nach Totalpräparat.

Fig. 15ั. Distomum clavigerum. Nach dem Leben.

Fig. 16. Distomum clavigerum. Ser. VI.

Fig. 17. Distomum clavigerum. Ser. IV.

Fig. 18. Distomum clavigerum. Ser. VI.

Fig. 19. Distomum endolobum.

Fig. 20. Distomum endolobum. Ser. VIII.

Fig. 21. Distomum endolobum. Ser. I.

Fig. 22. Distomum endolobum. Ser. II.

Fig. 23. Distomum endolobum. Ser. XVI 

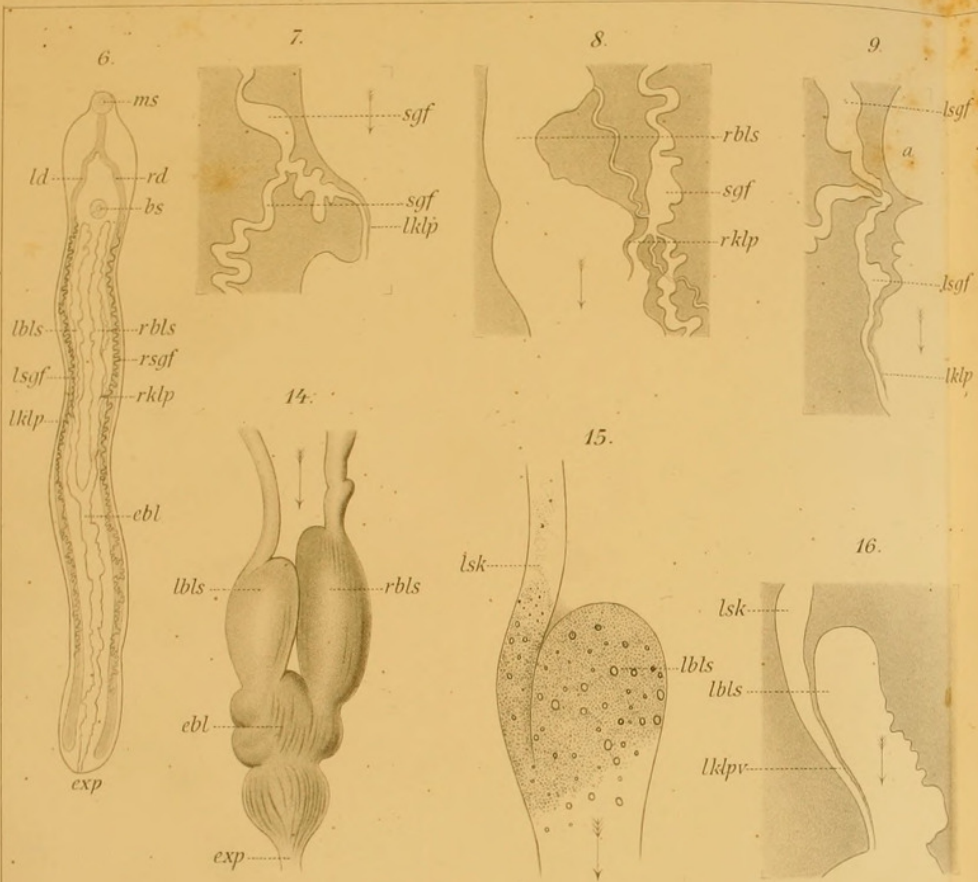

15.
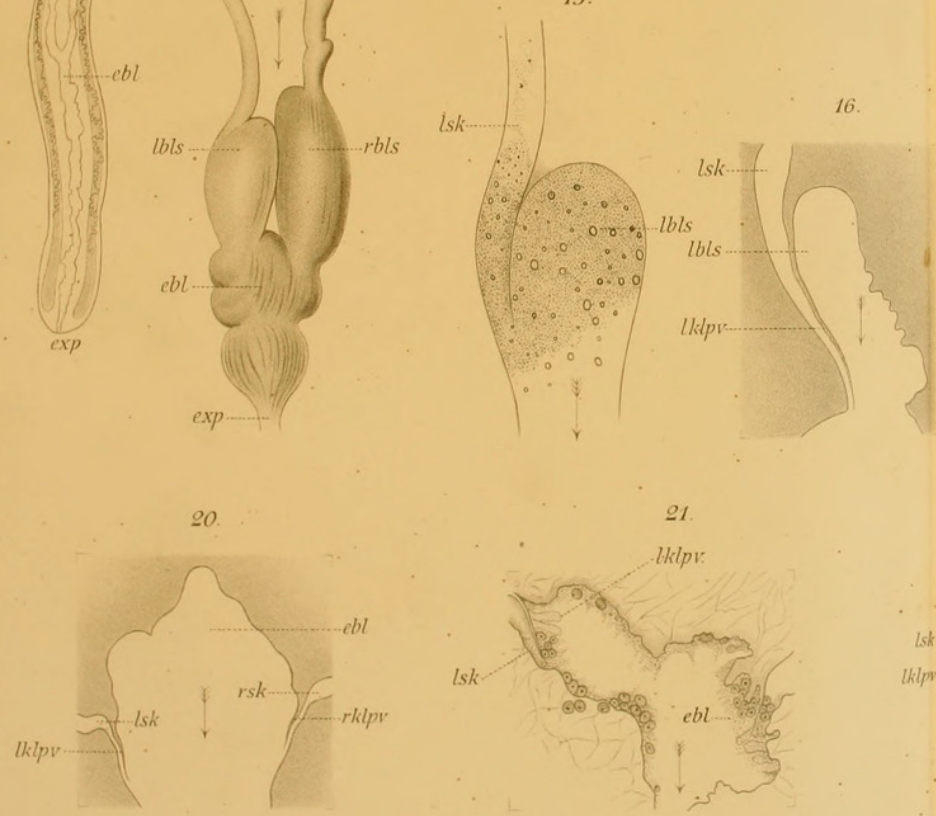

21.
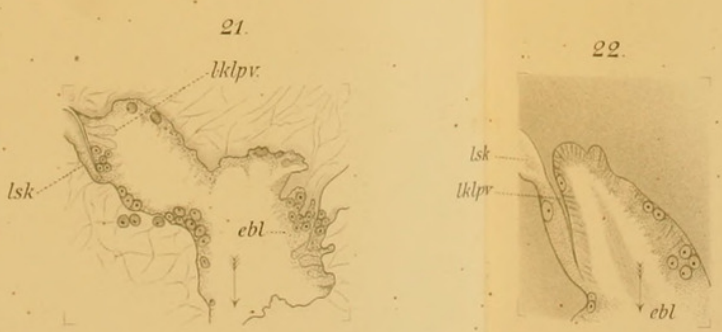

11

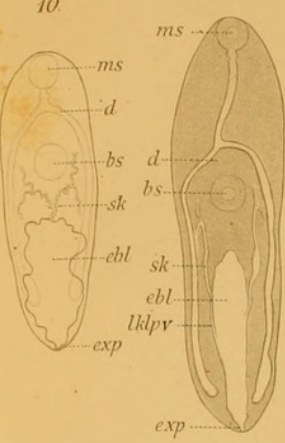

17.
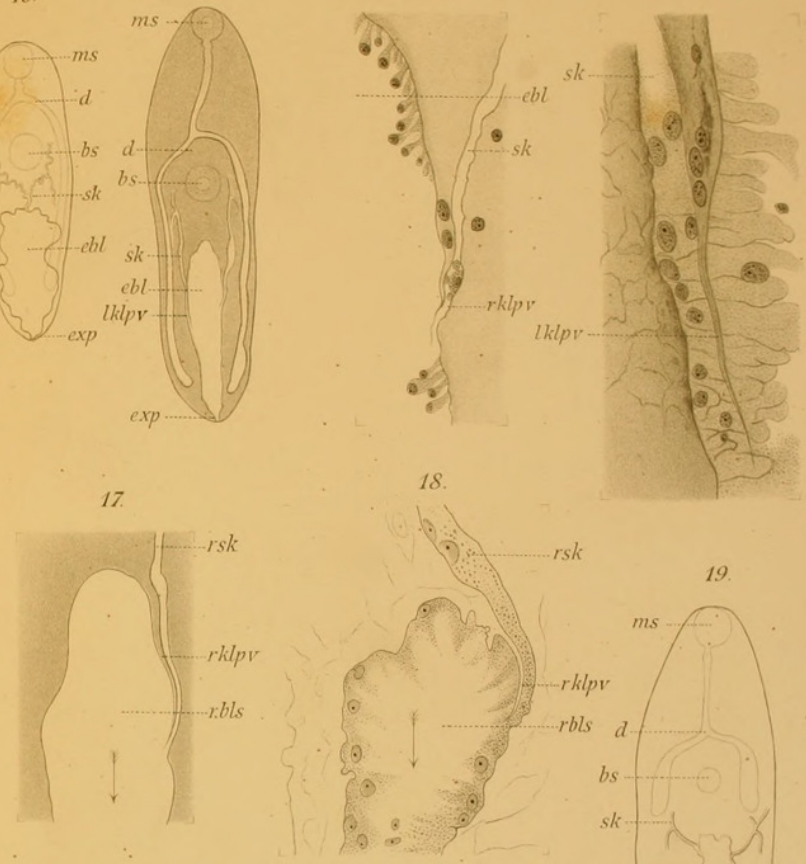

2.3
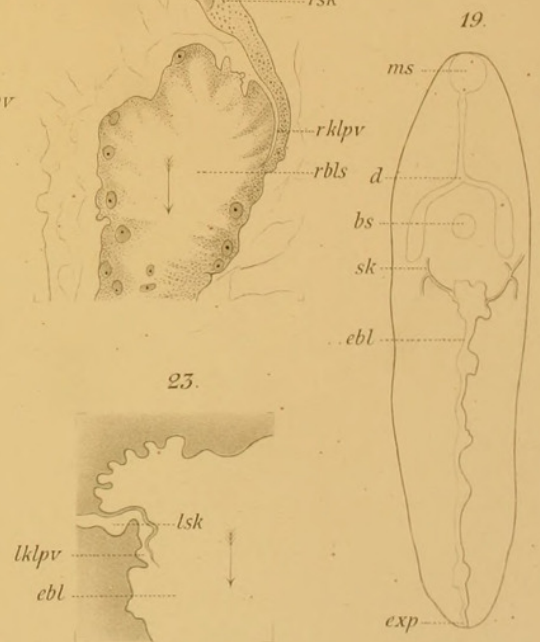

K. Kampmann. Trematoden 


\section{$2 \mathrm{BHL}$ Biodiversity Heritage Library}

Kampmann, Karl. 1894. "Ueber das Vorkommen von Klappenapparaten in den Excretionsorganen der Trematoden." Revue suisse de zoologie 2(4), 443-462. https://doi.org/10.5962/p.318229.

View This Item Online: https://www.biodiversitylibrary.org/item/38139

DOI: https://doi.org/10.5962/p.318229

Permalink: https://www.biodiversitylibrary.org/partpdf/318229

\section{Holding Institution}

MBLWHOI Library

\section{Sponsored by}

MBLWHOI Library

\section{Copyright \& Reuse}

Copyright Status: NOT_IN_COPYRIGHT

This document was created from content at the Biodiversity Heritage Library, the world's largest open access digital library for biodiversity literature and archives. Visit BHL at https://www.biodiversitylibrary.org. 